Annuaire du Collège de France 2017-2018

\title{
Médecine expérimentale
}

\section{Alain Fischer}

\section{OpenEdition}

Journals

Édition électronique

URL : https://journals.openedition.org/annuaire-cdf/15543

DOI : $10.4000 /$ annuaire-cdf. 15543

ISBN : 978-2-7226-0572-5

ISSN : 2109-9227

Éditeur

Collège de France

Édition imprimée

Date de publication : 30 décembre 2020

Pagination : 167-178

ISBN : 978-2-7226-0516-9

ISSN : 0069-5580

Référence électronique

Alain Fischer, "Médecine expérimentale », L'annuaire du Collège de France [En ligne], 118| 2020, mis en ligne le 01 avril 2021, consulté le 22 août 2022. URL : http://journals.openedition.org/annuaire-cdf/ 15543 ; DOI : https://doi.org/10.4000/annuaire-cdf.15543 


\title{
MÉDECINE EXPÉRIMENTALE
}

\author{
Alain FISCHER \\ Membre de l'Institut (Académie des sciences), \\ professeur au Collège de France
}

Mots-clés : médecine, immunité, immunologie, infection, génétique, virus

La série de cours et séminaires «Immunité anti-infectieuse (2) 》 est disponible, en audio et en vidéo, sur le site internet du Collège de France (https://www.college-defrance.fr/site/alain-fischer/course-2017-2018.htm).

\section{ENSEIGNEMENT}

COURS ET SÉMINAIRES - IMMUNITÉ ANTI-INFECTIEUSE (2)

\section{Introduction}

Nous avons poursuivi cette année l'exploration entreprise l'année passée des déterminants de l'immunité anti-infectieuse dans un certain nombre de pathologies microbiennes : infection par le virus d'immunodéficience humaine (VIH), infection par microorganismes intracellulaires dont la tuberculose, infection par le cytomégalovirus (CMV) et dans le contexte particulier des infections du système nerveux. Nous avons cherché à mettre en évidence la diversité des réponses immunes mises en jeu par l'analyse des modèles expérimentaux et des pathologies génétiques humaines ainsi que les stratégies d'échappement développées par les microorganismes au cours de l'évolution. Dès que possible, l'analyse des connaissances actuelles a servi de base de réflexion sur les possibilités d'interventions thérapeutiques et préventives (vaccination) en cours d'évaluation expérimentale ou clinique.

\section{Cours 1 - Le cerveau, un sanctuaire?}

Le système nerveux central est un organe précieux et fragile car très peu renouvelable. Il est l'objet de ce fait d'une protection particulière à l'égard des microorganismes à travers une barrière anatomique séparant circulation sanguine et 
parenchyme cérébral. Toutefois, il existe au sein de celui-ci des cellules résidentes qui représentent une première ligne de défense en cas d'infection : la microglie. De plus, l'ensemble des cellules du système nerveux dispose de capacité de réponse immune innée. En périphérie, dans les espaces périvasculaires et les lymphatiques périvasculaires circulent des lymphocytes et des cellules dendritiques mobilisables. Un grand nombre de microorganismes sont susceptibles de provoquer des infections des compartiments méningés, du système ventriculaire et du parenchyme responsable d'infections potentiellement sévères par les lésions irréversibles engendrées. Des pathogènes pénètrent soit comme hôte de cellules myéloïdes soit en ouvrant la jonction entre cellules endothéliales de la barrière hémato-encéphalique soit encore en infectant ces cellules. Au sein du système nerveux, les cellules de la microglie qui tapissent l'ensemble du parenchyme détectent les signaux de danger associés à la présence de microorganismes via les différentes voies de l'immunité innée. Sont ainsi rapidement produits des médiateurs pro-inflammatoires et chimioattractants de macrophages, polynucléaires et lymphocytes. On sait que des voies de l'immunité innée exercent un rôle déterminant dans le contrôle de certaines infections. Ainsi la voie induite par la reconnaissance d'acides nucléiques par le récepteur endosomal TLR3 est essentielle au contrôle de l'infection par le virus herpès simplex.

Une problématique majeure de la réponse immune antipathogène au sein du système nerveux concerne les conséquences délétères possibles de la réponse immune sur le système nerveux $(\mathrm{SN})$. On sait que beaucoup d'infections du $\mathrm{SN}$ - méningites ou encéphalites - sont responsables de séquelles graves en partie du fait de la réponse immunitaire, de la toxicité neuronale des cytokines et des produits de l'immunité innée (protéases, cytokines, radicaux oxygène). S'y ajoute la possible génération de réponses auto-immunes comme décrite au décours d'infection herpétique par production d'auto-anticorps dirigés contre des récepteurs synaptiques, possiblement par mimétisme moléculaire. Toutefois la microglie et les macrophages au décours du contrôle d'une infection produisent une série de molécules d'une part anti-inflammatoires et d'autre part impliquées dans les mécanismes de réparation : régénération axonale, remyélinisation. Cet équilibre subtil, dont la régulation est encore mal connue, est susceptible d'être mis à mal dans le développement de pathologies neurodégénératives.

\section{Séminaire 1 - Le méningocoque, cet illustre inconnu}

Docteur Sandrine Bourdoulous (DR CNRS, Institut Cochin, Paris), le 15 mai 2018

Sandrine Bourdoulous a rappelé l'importance en santé des méningites à méningocoque. Elle a exposé les résultats de sa recherche qui ont abouti à caractériser de façon précise les mécanismes moléculaires de l'invasion par le méningocoque à partir de sa capacité d'adhésion à l'endothélium vasculaire, sa capacité de prolifération et de franchissement de la barrière hémato-méningée.

\section{Cours 2 et 3 - L'infection par le VIH, une défaite du système immunitaire ?}

Le virus de l'immunodéficience humaine (VIH) est un lentivirus qui, en infectant les lymphocytes T CD4, est responsable du syndrome d'immunodéficience acquise (Sida) caractérisé par la survenue d'infections opportunistes, d'encéphalopathie et de tumeurs conduisant, en l'absence de traitement, au décès en quelques années. La 
biologie du virus, isolé en 1983 puis cloné en 1985, est aujourd'hui bien connue. Son mode d'entrée (récepteurs membranaires CD4 et récepteurs de chimiokines CCR5 ou CXCR4), l'intégration de son matériel génétique dans le génome de la cellule infectée après rétrotranscription de l'ARN génomique viral, les conditions de production de ces constituants, l'assemblage des virions et leurs productions ont été bien caractérisés. L'épidémiologie de l'infection est également bien connue. À ce jour, 37 millions de personnes sont infectées dans le monde, $95 \%$ vivent dans des pays pauvres, $50 \%$ sont des femmes et $9 \%$ des enfants. Deux millions de nouveaux cas surviennent chaque année. À ce jour, un peu plus de la moitié des patients sont traités par combinaison de chimiothérapie antirétrovirale, loin encore de l'objectif de l'OMS «90/90/90»: $90 \%$ de patients diagnostiqués dont $90 \%$ traités et $90 \%$ d'entre eux en rémission clinique. Les modalités de transmission du virus via les muqueuses ou le sang et les facteurs de risques associés ont été également bien déterminés. Lors de l'infection, il est observé une perte rapide de lymphocytes $\mathrm{T}$ CD4, directe par effet lytique de la réplication virale ou indirecte du fait de l'activation lymphocytaire chronique. Il est estimé qu'environ $10^{10}$ particules virales sont produites (et détruites par le système immunitaire) chaque jour expliquant une capacité de propagation de l'infection dans les tissus lymphoïdes muqueux et les organes lymphoïdes secondaires riches en lymphocytes T CD4. L'organisme cherche à limiter l'infection par la mise en jeu de multiples effecteurs de l'immunité : facteurs de restriction du cycle viral par l'immunité innée, à l'égard desquels le virus a développé des «contre-mesures", réponses adaptatives B et $\mathrm{T}$, notamment cytotoxiques dont on sait aujourd'hui que de la mise en jeu précoce et intense après le début de l'infection dépend la capacité de limiter l'infection comme discuté plus loin. Cette réponse n'est, pour l'essentiel, pas suffisante du fait de plusieurs facteurs critiques: a) infection et destruction des cellules impliquées dans les réponses immunes, b) taux de mutation élevé du virus du fait du caractère « infidèle » de la transcriptase reverse qui lui permet d'échapper aux réponses immunes par sélection de mutants. Ces caractéristiques rendent aussi compte à ce jour de l'échec des tentatives de protection par vaccination.

Deux grandes questions occupent aujourd'hui le champ de la recherche sur l'infection par le VIH : i) le concept de réservoir viral, et ii) le fait qu'un petit nombre de sujets infectés contrôlent la réplication du virus même en l'absence de traitement : les « elite controllers».

Le traitement antirétroviral permet de réduire considérablement la réplication virale et ainsi de restaurer un pool de lymphocytes T CD4 suffisant pour contrôler les risques infectieux mais il épargne un réservoir de cellules infectées de façon latente ou avec un taux de réplication virale faible. L'arrêt du traitement conduit à une reprise rapide de la réplication virale et à la réapparition du déficit immunitaire. Il est donc critique de comprendre les caractéristiques de ce réservoir afin d'essayer de le cibler par de nouvelles stratégies thérapeutiques. Ce réservoir concerne environ 1 lymphocyte $\mathrm{T} \mathrm{CD} 4 / 10^{6}$, capable de reprendre une réplication virale effective. $\mathrm{La}$ durée de vie de ce pool est de l'ordre de 44 mois, sa stabilité indiquant un équilibre entre perte cellulaire et division. On sait aujourd'hui qu'il est établi très tôt dans le cours de l'infection lors de la transition des lymphocytes $\mathrm{T}$ du stade effecteur à mémoire, soit après environ 6 à 12 jours d'infection au stade d'expression maximale du corécepteur du VIH CCR5. La capacité d'expansion (nécessaire au maintien du réservoir) est essentiellement le fait des capacités de prolifération homéostatique ou induite par un antigène, caractéristiques des cellules T mémoires. Diverses stratégies 
ont été envisagées pour détruire ce réservoir reposant sur l'activation de la réplication virale par des agents modificateurs épigénétiques suivies d'une immunothérapie ciblée par lymphocytes T CD8 cytotoxiques. Si cette approche a pu s'avérer efficace dans des modèles expérimentaux in vitro, les tentatives de mise en œuvre in vivo (souris humanisées) s'avèrent à ce jour un échec du fait de la résistance des lymphocytes infectés par sélection in vivo de résistance aux cellules cytotoxiques. Il faudrait pouvoir agir très précocement au cours de l'infection en maximisant la réponse immune cytotoxique au cours de l'établissement du réservoir.

Une petite fraction d'individus infectés par le VIH $(<1 \%)$ continue à contrôler la réplication virale après interruption du traitement antirétroviral et ce pendant des années ou des dizaines d'années. La compréhension de ce(s) mécanisme(s) de contrôle est bien sûr clé pour obtenir un tel effet chez tous les sujets infectés ! On sait que cette situation ne reflète pas de différence de souches virales infectantes. Les sujets « controllers » ont des caractéristiques génétiques (allèle HLA et KIR) qui contribuent (sans être rigoureusement indispensables) au fait que les réponses immunes $\mathrm{T}$ soient plus intenses, plus diversifiées - tant en matière d'Ag reconnus que de fonctions effectrices - et sans doute d'apparition plus précoce au cours de l'infection.

Néanmoins le contrôle de l'infection n'est pas complet chez ces sujets. Il persiste un certain degré de réplication virale au sein du compartiment de lymphocytes $\mathrm{T}$ présents dans les organes lymphoïdes secondaires dans les zones extra-folliculaires. On peut donc parler d'un phénomène de compartimentalisation du contrôle de l'infection.

Dans ce contexte, quelles nouvelles stratégies thérapeutiques envisager? Immunothérapie et vaccination doivent avoir pour objectif une génération rapide et intense de lymphocytes $\mathrm{T}$ cytotoxiques polyfonctionnels. L'utilisation d'anticorps neutralisants à large spectre - discutée dans mon cours il y a un an - peut représenter une forme d'immunothérapie passive, en cours d'évolution clinique. Les efforts de vaccination préventive ou thérapeutique n'ont pas à ce jour abouti à des résultats tangibles malgré la «sophistication» des protocoles intégrant l'utilisation de plusieurs antigènes, et l'adjonction d'une immunostimulation par cytokine.

Néanmoins, les progrès constants dans la compréhension de la génétique du réservoir viral et des réponses immunes efficaces des controllers devraient permettre de voir ce défi mieux relever au cours des prochaines années.

\section{Séminaire 2 - Comprendre la persistance du VIH, une nécessité pour guérir du Sida}

Docteur Monsef Benkirane (Institut de génétique humaine, CNRS Montpellier), le 22 mai 2018

Le docteur Benkirane a décrit ses travaux qui ont permis d'identifier une fraction des lymphocytes T CD4 du réservoir viral par l'expression membranaire de la molécule CD32 et de montrer qu'au sein de ces cellules il persiste à bas bruit un cycle réplicatif viral, ne permettant pas d'évoquer le caractère entièrement latent du réservoir. Ces travaux ouvrent des perspectives du ciblage thérapeutique de ces cellules. 


\section{Séminaire 3 - Comment induire une réponse immunitaire efficace contre le VIH ? Les leçons du VIH2}

Nicolas Manel (Institut Curie, Paris), le 29 mai 2018

Nicolas Manel a montré que la capacité de VIH2 par l'expression de la protéine Vpx permet d'inhiber le facteur de restriction de l'infection par le VIH SAMHD1 qui inhibe la transcriptase inverse par déprivation en deoxynucléotides nécessaires à la synthèse d' $A D N$ au sein des cellules dendritiques avec pour effet une amplification de la réponse immune $\mathrm{T}$ par les $\mathrm{Ag}$ de VIH présentes par les cellules dendritiques. $A$ contrario, HIV1 qui ne possède pas d'élément Vpx dans son génome peut persister « caché » au sein des cellules dendritiques et contribuer à la persistance du réservoir.

\section{Cours 4 - Immunité contre les microorganismes à développement intracellulaire}

Ce cours, le suivant ainsi que les deux séminaires qui les accompagnent (voir infra) porte sur les particularités des infections par les microorganismes à développement intracellulaire et des modalités de la réponse immune. Les cas de quatre microorganismes ont été discutés : les bactéries Salmonella, mycobactéries, Brucella et Listeria.

Les salmonelles sont des bactéries à Gram négaif, parasites facultatifs des macrophages. Elles provoquent, en fonction des types (typhi/paratyphi et non typhi), respectivement la fièvre typhoïde ou des pathologies infectieuses intestinales. La salmonelle typhi est endocytée par les cellules M de l'épithélial intestinal et les cellules épithéliales elles-mêmes. S'ensuit une dissémination extracellulaire et l'infection (par phagocytose) des macrophages et cellules dendritiques associées au système immunitaire de l'intestin. Les réponses immunes impliquent l'immunité innée via les récepteurs des familles TLR et NLR (inflammasome). Ces derniers sont activés par des produits bactériens dont la flagelline dans le cytosol des cellules épithéliales. En plus de la réponse inflammatoire, l'inflammasome conduit à l'expulsion dans la lumière intestinale des cellules infectées. Des modèles d'infection expérimentale chez la souris ont démontré l'importance de ce mécanisme de défense dans le contrôle de l'infection par Salmonelle. L'immunité adaptative est également mise en jeu conduisant à l'induction de mécanismes de mort cellulaire de Salmonella au sein de macrophages via la sécrétion de l'interféron $\gamma$ par les lymphocytes TH1 et le recrutement de polynucléaires neutrophiles par l'IL17 produite par les lymphocytes TH17. Des modèles expérimentaux ont là aussi démontré le caractère essentiel des lymphocytes $\mathrm{T}$ et de ces populations dans le contrôle de l'infection. Chez l'homme, le défaut génétique en IL12p40 ou en chaîne b1 du récepteur de l'IL12 provoque (entre autres) une prédisposition aux infections par Salmonelle du fait du défaut conjoint d'activation des lymphocytes TH1 (production d'interféron $\gamma$ ) et TH17.

Un élément essentiel de la protection contre l'infection par Salmonelle consiste en la compétition avec la flore intestinale commensale. Il a été récemment montré que la flore de souris adulte (mais pas de nouveaux-nés) protégeait contre l'infection par Salmonelle grâce à la présence de Clostridium qui crée un milieu défavorable à Salmonella. Comme tous les microorganismes, Salmonella a développé au cours de la coévolution avec l'homme des facteurs de résistance multiples aux effecteurs des immunités innées et adaptatives. Salmonella peut «profiter » du transfert horizontal par infection par un phage qui confère un facteur de virulence. La réponse 
inflammatoire paradoxalement favorise la production du phage et le transfert propice à l'acquisition de la virulence. Un des intérêts de l'étude de la réponse immune à Salmonella est d'aborder l'hypothèse du rôle de l'infection chronique comme facteur d'induction d'une pathologie inflammatoire chronique de l'intestin. Expérimentalement, un mécanisme moléculaire susceptible de rendre compte d'un tel lien a été mis en évidence.

\section{Séminaire 4 - Can we take advantage of Brucella virulence factors?}

\section{Jean-Pierre Gorvel (DR CNRS CIML Marseille Luminy), le 5 juin 2018}

Jean-Pierre Gorvel a décrit ses travaux ayant trait à la caractérisation de facteurs de virulence de Brucella, bactérie responsable de la brucellose chez l'homme mais aussi chez les ruminants. Il a montré comment ces facteurs peuvent être utilisés comme agent d'immunisation pour la mise au point de vaccin efficace capable de prévenir cette infection en médecine vétérinaire, un objectif à l'impact économique loin d'être négligeable.

\section{Cours 5 - Le système immunitaire face à une infection disséminée dans l'organisme. Mycobacterium tuberculosis (MT)}

MT est l'agent de la tuberculose, bacille complexe qui coévolue avec l'homme depuis au moins 70000 ans. La tuberculose a entraîné environ un milliard de décès au cours des 200 dernières années. On estime qu'environ plus de 9 millions de personnes sont infectées chaque année dont $13 \%$ dans le contexte d'une co-infection avec le VIH. Le $\mathrm{XX}^{\mathrm{e}}$ siècle a été marqué par une régression de l'incidence de l'infection grâce à l'amélioration des conditions de vie, du diagnostic, des traitements à partir de 1950 et dans une moindre mesure de la vaccination. Le développement de formes multirésistantes de MT aux agents antimycobactériens (environ 500000 cas par an dans le monde) est un sujet de préoccupation. L'histoire naturelle de la tuberculose est marquée par le fait que seulement $10 \%$ des sujets infectés développent une tuberculose maladie, chez $90 \%$ l'infection reste latente. La lésion primitive consiste en un granulome formé de macrophages infectés par MT entourés de cellules immunitaires recrutées : cellules dendritiques, lymphocytes $\mathrm{T}$ et $\mathrm{B}$. Le granulome peut évoluer vers son ouverture et la dissémination du matériel nécrotique présent en son centre provoquant la propagation de la maladie ; de façon alternative le granulome peut conduire à la stérilisation de la lésion ou à l'endiguement durable des MT qu'il contient. Ce sont les macrophages infectés qui sécrètent des facteurs de recrutement (chimiokines) et d'activation des lymphocytes $\mathrm{T}$ (et B). La production d'interféron $\gamma$ par les lymphocytes TH1 confère aux macrophages infectés la capacité de tuer MT par la mise en jeu de plusieurs mécanismes dont la production de NO (oxyde nitrique). Les anomalies héréditaires du système immunitaire nous enseignent que la production de radicaux oxygène libérés par les macrophages et l'axe IL-12 interféron $\gamma$ sont indispensables au contrôle des infections par les mycobactéries, leurs défauts étant responsables d'infections disséminées par les mycobactéries même par celles qui ne possèdent pas les facteurs de virulence de MT.

Une question majeure de la prise en charge des patients infectés par MT est la prédiction de l'évolutivité de l'infection. Une étude a permis d'établir que le profil d'expression de gènes de réponse à l'interféron de type 1 permettrait dans une 
certaine mesure de prédire la progression de la maladie, ce qui pourrait servir de base à une intervention thérapeutique ciblée. MT a sélectionné au cours de l'évolution de nombreuses mutations résultant dans une capacité de résistance aux réponses immunes, de l'inhibition de la fusion phagosome-liposome, à l'inhibition de la présentation antigénique aux lymphocytes T, l'inhibition de l'autophagie, à l'induction d'effets suppresseurs paradoxaux des polynucléaires neutrophiles et d'autres cellules myéloïdes, et aux effets facilitateurs de l'infection de l'interféron de type 1 . Ces facteurs de virulence (résistance) ont été pour l'essentiel caractérisés sur le plan moléculaire.

La vaccination par le BCG, MT vivant atténué a été introduite il y a déjà plus de 90 ans. Ce vaccin protège partiellement des tuberculoses du nourrisson et des enfants mais pas de la tuberculose pulmonaire. En particulier ce vaccin n'induit pas de réponse $\mathrm{T}$ CD8. De nombreuses tentatives de modification du BCG par recombinaison génétique sont testées avec notamment l'objectif de permettre la présentation croisée d'antigènes par les cellules dendritiques aux lymphocytes T CD8. Des essais cliniques sont en cours mais il est encore trop tôt pour en évaluer l'efficacité. Il n'est pas exclu qu'il faille effectuer encore d'autres modifications fondées sur une meilleure connaissance des facteurs de virulence de MT et des réponses immunes pour obtenir un vaccin pleinement efficace.

\section{Séminaire 5 - Listeria monocytogenes, un envahisseur silencieux}

Marc Lecuit (université Paris Descartes, hôpital Necker, Institut Pasteur, Paris), le 12 juin 2018

Listeria monocytogenes est une autre bactérie à développement intracellulaire responsable d'infections graves du système nerveux central chez le nouveau-né, les personnes âgées et les sujets immunodéficients. Marc Lecuit a décrit ses travaux qui ont conduit à caractériser les mécanismes d'invasion par Listeria au niveau de l'intestin, sa capacité de dissémination et d'infection du système nerveux central en en identifiant les facteurs.

\section{Cours 6 - Le cytomégalovirus, un vieil ennemi à combattre. Comment ?}

Le cytomégalovirus (CMV) est un virus à ADN double brin qui fait partie de la famille des herpèsvirus (HHV5). Il est responsable d'une maladie bénigne ou asymptomatique dans l'immense majorité des cas. En revanche, il provoque une maladie sévère chez le fotus et les personnes immunodéprimées. Ce virus est persistant et sa réplication est sporadiquement réactive. Environ $60 \%$ de l'humanité est infectée par le CMV. La contamination se produit par les sécrétions. Cellules épithéliales, endothéliales, monocytes et macrophages en sont les cibles cellulaires principales. Il est probable que l'infection latente se produise au sein des monocytes et des progéniteurs hématopoḯtiques. Parmi les cellules impliquées dans la réponse immune anti-CMV, il convient d'évoquer le rôle des lymphocytes Natural Killer (NK) qui sont activés en particulier par la reconnaissance de la molécule HLA E associée sans doute à un peptide stabilisant issu du CMV. NKG2C est le récepteur activateur concerné. D'autres signaux concourent à l'activation des cellules NK, ces dernières agissent en tant qu'effecteurs de l'immunité anti-CMV par production d'interféron $\gamma$ et cytotoxicité des cellules infectées. Les lymphocytes T exercent une 
fonction essentielle de protection contre CMV en particulier par leur fonction cytotoxique. Les formes sévères d'infection à CMV chez des sujets transplantés peuvent être ainsi contrôlées par immunothérapie adoptive consistant en des lymphocytes T anti-CMV. La détection dans le sang de lymphocytes T anti-CMV à l'aide de tétramères montre que ces cellules représentent une fraction importante de la population $\mathrm{T}$ (de l'ordre de 9 à $10 \%$ des $\mathrm{T}$ mémoires) indiquant la place de l'infection chronique par CMV (épisodes de réactivation) dans l'équilibre des réponses immunitaires. L'accumulation de ces cellules peut s'observer sous forme de lymphocytes $\mathrm{T}$ «épuisés » chez les personnes âgées, d'efficacité moindre à l'égard de l'infection par le CMV. Les mécanismes moléculaires responsables de ce phénomène, observés dans d'autres infections virales chroniques commencent à être identifiés. Il a été de plus montré que le fait d'être porteur du CMV modifie significativement l'architecture du système immunitaire comme observé en comparant des jumeaux vrais discordants pour l'infection à CMV. Sont principalement concernés les lymphocytes $\mathrm{T} \gamma / \delta$, en T CD8 effecteurs et les polynucléaires neutrophiles. De façon surprenante, il a été montré chez l'homme et la souris que l'infection chronique par le CMV favorise l'efficacité de la vaccination antigrippale chez les adultes jeunes, via une immunité « hétérologue », conférée par la production d'interféron $\gamma$ induite par l'infection chronique à CMV. Néanmoins, il est également possible que l'infection chronique à CMV mal contrôlée (voir supra) soit délétère chez le sujet âgé du fait en particulier de l'infection chronique de cellules endothéliales, l'inflammation engendrée et un risque accru de pathologies vasculaires. L'infection par le CMV représente un modèle illustratif des conséquences possibles d'une infection chronique sur l'équilibre du système immunitaire en général.

\section{Séminaire 6 - Le cytomégalovirus peut-il nous aider à comprendre les lymphocytes T de type $\gamma / \delta$ ?}

Julie Dechanet-Merville (CNRS, université de Bordeaux), le 19 juin 2018

Julie Dechanet-Merville a montré l'importance de la réponse de sous-populations de lymphocytes $\mathrm{T} \gamma / \delta$ au cours de l'infection par le CMV en particulier chez les sujets transplantés. Elle a caractérisé ces populations et cherche aujourd'hui à identifier les ligands activateurs afin d'envisager de développer des stratégies d'immunothérapie dans le cadre de l'infection par le CMV mais aussi dans d'autres contextes d'activation des lymphocytes $\mathrm{T} \gamma / \delta$ comme certains cancers.

\section{RECHERCHE}

Nous avons poursuivi l'étude des caractéristiques globales des anomalies génétiques communes du système immunitaire et montré la fréquence de mutations gain de fonction de gènes de l'immunité innée responsable de pathologies autoimmunes et/ou inflammatoires. À l'inverse, les anomalies pertes de fonction régulatrices de l'immunité adaptative sont plus souvent responsables de maladies auto-immunes. Ces données suggèrent qu'au cours de l'évolution a été sélectionné un système d'immunité innée robuste et diversifié relativement peu soumis à régulation à l'opposé du système immunitaire adaptatif moins robuste mais soumis à de multiples contrôles tant le risque de réponse contre les constituants du soi sont 
élevés. Nous avons participé à la caractérisation de deux nouvelles anomalies héréditaires responsables de défaut de fonction des lymphocytes $\mathrm{T}$ et $\mathrm{B}$ : mutation $I K Z F 1$ et $R A S G R P 1$. La seconde provoque un défaut d'expansion des lymphocytes $\mathrm{T}$ lors de la stimulation antigénique responsable d'un contrôle défectueux d'infections virales. Cette observation démontre le rôle clé de la voie RAS/MAP kinase dans l'induction de la prolifération des lymphocytes T, activation qui entre autres résulte dans l'induction de l'expression de la cytidine triphosphate synthétase 1 nécessaire à l'adaptation métabolique des lymphocytes en division. D'autres études physiopathologiques ont été menées concernant les cellules T régulatrices au cours du syndrome lymphoprolifératif auto-immun par déficit en FAS et le rôle de l'inflammation au cours de la granulomatose septique chronique.

Sur le plan clinique des descriptions de cohortes concernant le syndrome d'Evans et les mutations gain de fonction de la PI3 kinase ont été effectuées. Des procédures diagnostiques génétiques ont été développées pour la mise en évidence des anomalies de génération du répertoire $\mathrm{T}$ et des pathologies inflammatoires de l'intestin. L'efficacité de l'allogreffe de cellules souches hématopoïétiques comme traitement de la protéinose alvéolaire par déficit en récepteur du GM-CSF a été pour la première fois rapportée. Nous avons enfin mené 1) une étude épidémiologique sur la prévalence des cas de déficits immunitaires héréditaires (DIH) par l'enquête systématique auprès de tous les médecins et pharmaciens dans seize départements de la métropole et 2) une étude de la qualité de vie des enfants atteints de DIH.

\section{PuBLications}

FISCHER A., « Gene therapy: From birth to maturity requires commitment to science and ethics », Human Gene Therapy, vol. 28, no 11, 2017, p. 958, DOI : 10.1089/hum.2017.29053.afr.

Garcelon N., Neuraz A., Benoit V., Salomon R., Kracker S., Suarez F., BahiBuisson N., HAdj-RABia S., Fischer A., MunNich A. et Burgun A., «Finding patients using similarity measures in a rare diseases-oriented clinical data warehouse: Dr. Warehouse and the needle in the needle stack », Journal of Biomedical Informatics, vol. 73, 2017, p. 5161, DOI : 10.1016/j.jbi.2017.07.016.

Gabrion A., Hmitou I., Moshous D., Neven B., Lefevre-Utile A., Diana J.S., Suarez F., Picard C., Blanche S., Fischer A., Cavazzana M. et Touzot F., « Mammalian target of rapamycin inhibition counterbalances the inflammatory status of immune cells in patients with chronic granulomatous disease », The Journal of Allergy and Clinical Immunology, vol. 139, $\mathrm{n}^{\mathrm{o}}$ 5, 2017, p. 16411649.e6, DOI : 10.1016/j.jaci.2016.08.033.

Klemann C., Esquivel M., Magerus-Chatinet A., Lorenz M.R., Fuchs I., Neveux N., Castelle M., Rohr J., Da Cunha C.B., Ebinger M., Kobbe R., Kremens B., Kollert F., Gambineri E., Lehmberg K., Seidel M.G., Siepermann K., Voelker T., Schuster V., Goldacker S., Schwarz K., Speckmann C., Picard C., Fischer A., RieuX-Laucat F., EHL S., RENSING-EHL A. et NEVEN B., « Evolution of disease activity and biomarkers on and off rapamycin in 28 patients with autoimmune lymphoproliferative syndrome », Haematologica, vol. 102, no 2, 2017, e52-e56, DOI : 10.3324/haematol.2016.153411.

Mahlaoui N., Jais J.-P., Brosselin P., Mignot C., Beaurain B., Brito C., Costes L., Courteille V., De Vergnes N., Alcaïs A., Fischer A. et collaborators CEREDIH, « Prevalence of primary immunodeficiencies in France is underestimated », The Journal of Allergy and Clinical Immunology, vol. 140, $\mathrm{n}^{\mathrm{O}}$ 6, 2017, p. 17311733, DOI : 10.1016/j. jaci.2017.06.020. 
Mao H., Yang W., Latour S., Yang J., Winter S., Zheng J., Ni K., Lv M., LiU C., HuAng H., Chan K.-W., PUi-Wah LeE P., Tu W., Fischer A. et LAU Y.-L., «RASGRP1 mutation in autoimmune lymphoproliferative syndrome-like disease », The Journal of Allergy and Clinical Immunology, 2017, DOI : 10.1016/j.jaci.2017.10.026.

Adam F., Kauskot A., Kurowska M., Goudin N., Munoz I., Bordet J.-C., Huang J.-D., Bryckaert M., Fischer A., Borgel D., De SAint Basile G., Christophe O.D. et MÉNASCHÉ G., « Kinesin-1 is a new actor involved in platelet secretion and thrombus stability », Arteriosclerosis, Thrombosis, and Vascular Biology, vol. 38, $\mathrm{n}^{\circ}$ 5, 2018, p. 1037-1051, DOI : 10.1161/ATVBAHA.117.310373.

Barlogis V., Mahlaoui N., Auquier P., Fouyssac F., Pellier I., Vercasson C., Allouche M., De Azevedo C.B., Moshous D., Neven B., Pasquet M., Jeziorski E., Aladjidi N., Thomas C., Gandemer V., Mazingue F., Picard C., Blanche S., Michel G. et FISCHER A., "Burden of poor health conditions and quality of life in 656 children with primary immunodeficiency », The Journal of Pediatrics, vol. 194, 2018, p. 211-217.e5, DOI : 10.1016/j.jpeds.2017.10.029.

Berland A., Rosain J., Kaltenbach S., Allain V., Mahlaoui N., Melki I., Fievet A., Dubois d'Enghien C., Ouachée-Chardin M., Perrin L., Auger N., Cipe F.E., Finocchi A., Dogu F., Suarez F., Moshous D., Leblanc T., Belot A., Fieschi C., Boutboul D., Malphettes M., Galicier L., OKsenhendler E., Blanche S., Fischer A., Revy P., StOPPA-LYONNET D., PICARD C. et DE VILlaRTAY J.-P., «PROMIDIS $\alpha$ : a TCR $\alpha$ signature associated with immunodeficiencies caused by V(D)J recombination defects », The Journal of Allergy and Clinical Immunology, 2018, DOI : 10.1016/j.jaci.2018.05.028.

Besnard C., Levy E., Aladjidi N., Stolzenberg M.-C., Magerus-Chatinet A., Alibeu O., Nitschke P., Blanche S., Hermine O., Jeziorski E., LANDMAN-PARKer J., Leverger G., Mahlaoui N., Michel G., Pellier I., Suarez F., Thuret I., De SaintBasile G., Picard C., Fischer A., Neven B., RieuX-Laucat F. et Quartier P., « Pediatriconset Evans syndrome: Heterogeneous presentation and high frequency of monogenic disorders including LRBA and CTLA4 mutations », Clinical Immunology, vol. 188, 2018, p. 5257, DOI : 10.1016/j.clim.2017.12.009.

Boutboul D., Kuehn H.S., Wyngaert Z.V. de, Niemela J.E., Callebaut I., Stoddard J., Lenoir C., Barlogis V., Farnarier C., Vely F., Yoshida N., Kojima S., Kanegane H., Hoshino A., Hauck F., Lhermitte L., Asnafi V., Roehrs P., Chen S., Verbsky J.W., Calvo K.R., Husami A., Zhang K., Roberts J., Amrol D., Sleaseman J., Hsu A.P., Holland S.M., Marsh R., Fischer A., Fleisher T.A., PicArd C., LATOUR S. et RosENZWEIG S.D., « Dominant-negative IKZF1 mutations cause a T, B, and myeloid cell combined immunodeficiency », The Journal of Clinical Investigation, vol. 128, $\mathrm{n}^{\mathrm{o}} 7,2018$, p. 30713087, DOI : 10.1172/JCI98164.

Charbit-Henrion F., Parlato M., Hanein S., Duclaux-Loras R., Nowak J., Begue B., Rakotobe S., Bruneau J., Fourrage C., Alibeu O., RieuX-Laucat F., Lévy E., Stolzenberg M.-C., Mazerolles F., latour S., Lenoir C., Fischer A., Picard C., Aloi M., Amil Dias J., Ben Hariz M., Bourrier A., Breuer C., Breton A., Bronski J., Buderus S., Cananzi M., Coopman S., CrémilleuX C., Dabadie A., Dumant-Forest C., Egritas Gurkan O., Fabre A., Fischer A., German Diaz M., GonZalez-Lama Y., Goulet O., Guariso G., Gurcan N., Homan M., Hugot J.-P., Jeziorski E., Karanika E., Lachaux A., Lewindon P., Lima R., Magro F., Major J., Malamut G., Mas E., Mattyus I., Mearin L.M., MeleK J., NaVAs-Lopez V.M., PaerregaArd A., Pelatan C., Pigneur B., Pinto Pais I., Rebeuh J., Romano C., Siala N., Strisciuglio C., TempiaCaliera M., Tounian P., Turner D., Urbonas V., Willot S., Ruemmele F.M. et CerfBENSUSSAN N., «Diagnostic yield of next-generation sequencing in very early-onset inflammatory bowel diseases: A multicenter study », Journal of Crohn's and Colitis, 2018, DOI : 10.1093/ecco-jcc/jjy068. 
FISCHER A. et RAUSEll A., "What do primary immunodeficiencies tell us about the essentiality/redundancy of immune responses? », Seminars in Immunology, vol. 36, 2018, p. 1316, DOI : 10.1016/j.smim.2017.12.001.

Frémond M.-L., Hadchouel A., Schweitzer C., Berteloot L., Bruneau J., Bonnet C., Cros G., Briand C., Magnani A., Pochon C., Delacourt C., Cavazzana M., Moshous D., Fischer A., Blanche S., BliC J.D. et NeVEn B., « Successful haematopoietic stem cell transplantation in a case of pulmonary alveolar proteinosis due to GM-CSF receptor deficiency », Thorax, vol. 73, no 6, 2018, p. 590592, DOI : 10.1136/thoraxjnl-2017-211076.

Maccari M.E., Abolhassani H., Aghamohammadi A., Aiuti A., Aleinikova O., Bangs C., Baris S., Barzaghi F., BaXendale H., Buckland M., Burns S.O., Cancrini C., Cant A., Cathébras P., Cavazzana M., Chandra A., Conti F., Coulter T., Devlin L.A., Edgar J.D.M., Faust S., Fischer A., Prat M.G., Hammarström L., HeEg M., Jolles S., Karakoc-Aydiner E., Kindle G., Kiykim A., Kumararatne D., Grimbacher B., Longhurst H., Mahlaoui N., Milota T., Moreira F., Moshous D., Mukhina A., Neth O., Neven B., Nieters A., Olbrich P., Ozen A., Schmid J.P., Picard C., Prader S., Rae W., Reichenbach J., Rusch S., Savic S., Scarselli A., Scheible R., Sediva A., Sharapova S.O., Shcherbina A., Slatter M., Soler-Palacin P., Stanislas A., Suarez F., TuCCi F., Uhlmann A., VAn MontFrans J., Warnatz K., Williams A.P., Wood P., Kracker S., CondlifFe A.M. et EHL S., «Disease evolution and response to rapamycin in activated phosphoinositide 3-kinase $\delta$ syndrome: The European society for immunodeficiencies-activated phosphoinositide 3-kinase $\delta$ syndrome registry », Frontiers in Immunology, vol. 9, 2018, p. 543, DOI : 10.3389/fimmu.2018.00543.

Mazerolles F., Stolzenberg M.-C., Pelle O., Picard C., Neven B., Fischer A., MAgERUS-Chatinet A. et RiEUX-LAUCAT F., « Autoimmune lymphoproliferative syndromeFAS patients have an abnormal regulatory $\mathrm{T}$ cell (Treg) phenotype but display normal natural Treg-suppressive function on T cell proliferation », Frontiers in Immunology, vol. 9, 2018, p. 718, DOI : 10.3389/fimmu.2018.00718.

Winter S., Martin E., Boutboul D., Lenoir C., BoudjemaA S., Petit A., Picard C., FISCHER A., LEVERGER G. et LATOUR S., «Loss of RASGRP1 in humans impairs T-cell expansion leading to Epstein-Barr virus susceptibility », EMBO Molecular Medicine, vol. 10, $\mathrm{n}^{\mathrm{o}} 2$ 2, 2018, p. 188199, DOI : 10.15252/emmm.201708292.

Barzaghi F., Hernandez L.C.A., Neven B., Ricci S., Kucuk Z.Y., Bleesing J.J., Nademi Z., Slatter M.A., Ulloa E.R., Shcherbina A., Roppelt A., Worth A., Silva J., Aiuti A., Murguia-Favela L., Speckmann C., Carneiro-Sampaio M., Fernandes J.F., Baris S., Ozen A., Karakoc-Aydiner E., Kiykim A., Schulz A., Steinmann S., Notarangelo L.D., Gambineri E., Lionetti P., SheArer W.T., Forbes L.R., MARTinez C., Moshous D., Blanche S., Fisher A., Ruemmele F.M., Tissandier C., OuACheEChardin M., RieuX-Laucat F., Cavazzana M., QAsim W., LuCarelli B., Albert M.H., Kobayashi I., Alonso L., De Heredia C.D., Kanegane H., LawitschKa A., Seo J.J., GonZalez-Vicent M., Diaz M.A., Goyal R.K., Sauer M.G., YesilipeK A., Kim M., YilmaZ-Demirdag Y., Bhatia M., KhleVner J., Padilla E.J.R., Martino S., Montin D., Neth O., Molinos-Quintana A., Valverde-Fernandez J., Broides A., Pinsk V., Ballauf A., Haerynck F., Bordon V., Dhooge C., Garcia-Lloret M.L., Bredius R.G., Kawak K., Haddad E., Seidel M.G., Duckers G., Pai S.-Y., Dvorak C.C., Ehl S., Locatelli F., Goldman F., Gennery A.R., Cowan M.J., RonCAROlo M.-G. et BACCHETTA R., «Long-term follow-up of IPEX syndrome patients after different therapeutic strategies: An international multicenter retrospective study », Journal of Allergy and Clinical Immunology, vol. 141, no 3, 2018, 1036-1049.e5, DOI : 10.1016/j.jaci.2017.10.041. 
